\title{
Quasi-optics-inspired low-profile endfire antenna element
}

\author{
Vedaprabhu Basavarajappa ${ }^{1,2^{*}}$ (D), Beatriz Bedia Exposito ${ }^{1}$, Lorena Cabria ${ }^{1}$ and Jose Basterrechea ${ }^{2}$
}

\begin{abstract}
In this paper, the design, operational principle and experimental validation of an endfire antenna element that is inspired by the energy-focusing characteristics of graded-index optical fibre are presented. The antenna operates with a bandwidth of $1.5 \mathrm{GHz}$ centred around $14 \mathrm{GHz}$. It has a gain of around $5.5 \mathrm{dBi}$ along the band of interest and a good pattern stability over the band. The antenna derives its unique nature from the arrangement of the arc-shaped parasitics, that couple onto the antenna's driver dipole. An electromagnetic refractive index retrieval mechanism is used to guide the placement of the parasitics to enhance the gain. In addition to the design principle, a parametric study of the main parameters and their influence on the antenna behaviour is presented. The antenna is a potential candidate for use in multi-user massive MIMO antenna arrays for $5 \mathrm{G}$ communications where space is premium and in antenna array applications where a low-profile antenna element with a high gain is a necessity.
\end{abstract}

\section{Introduction}

Scenarios of high data-rate transmissions of several gigabits per second require the use of wide bandwidth and high directivity beams. The wide bandwidth is attained by shifting to a higher millimetre wave frequency, and to obtain high directivity beam, antennas need to be designed to focus the beam with high front-to-back (F/B) ratio. When employed in an array, such an antenna further enhances the directivity offered from the array. Recently there has been an increasing interest in the $14-15-\mathrm{GHz}$ band [1-3]. A printed antenna configuration is attractive owing to the simplicity of manufacturing, scalability and the reduced cost. The conventional patch or printed dipole antennas generally have a low directivity omnidirectional pattern. Among the techniques used to enhance this directivity, the use of parasitic elements coupled to the printed dipole is appealing. Accordingly, an endfire antenna element based on parasitics aided quasi-Yagi and fibre-optics principle is proposed in this paper. Following the analogy to the graded refractive index optical fibre energy-focusing principle, the designed antenna element has its gain and

\footnotetext{
* Correspondence: veda@ieee.org

'Department of Antennas, TTI Norte, Parque Científico y Tecnológico de Cantabria, C/ Albert Einstein n 14, 39011 Santander, Spain

${ }^{2}$ Departamento de Ingeniería de Comunicaciones, Universidad de Cantabria, Av. de los Castros s/n, 39005 Santander, Spain
}

directivity enhanced-mainly by a unique arrangement of parasitics around the driving sources, aided by reconfigured refractive index-referred to as quasioptic approach.

Endfire antenna topologies such as the helical antenna, antipodal Vivaldi antenna and the Yagi-Uda antenna have been used in applications such as ultrawideband, phased array, radars and microwave imaging. Printed endfire antenna elements have been used for high-gain applications. These are generally fed by a half wavelength resonant driver, and the coupling between the driver element and the director element is set to maximize the achievable gain. The compactness of resonant type antennas and the broad bandwidth characteristics were both simultaneously achieved in a single Yagi antenna [4] where a microstrip to CPS transition balun was used to add to the profile length. With the compactness and broad bandwidth addressed by antennas in $[4,5]$, papers $[6,7]$ focus on improving the antenna gain using techniques of a microstrip-fed inset patch as the driver, with parasitic patches added as directors to enhance the gain. The dipole feeding point in this antenna design is based upon [8], where the antenna operates in the $2-4-\mathrm{GHz}$ band with a gain of around $5 \mathrm{dBi}$ over the band. Since Yagi-Uda antennas or the endfire antennas in general are protrude-out-ofwall configurations, it adds immensely if this dimension of profile length could be minimized while retaining 


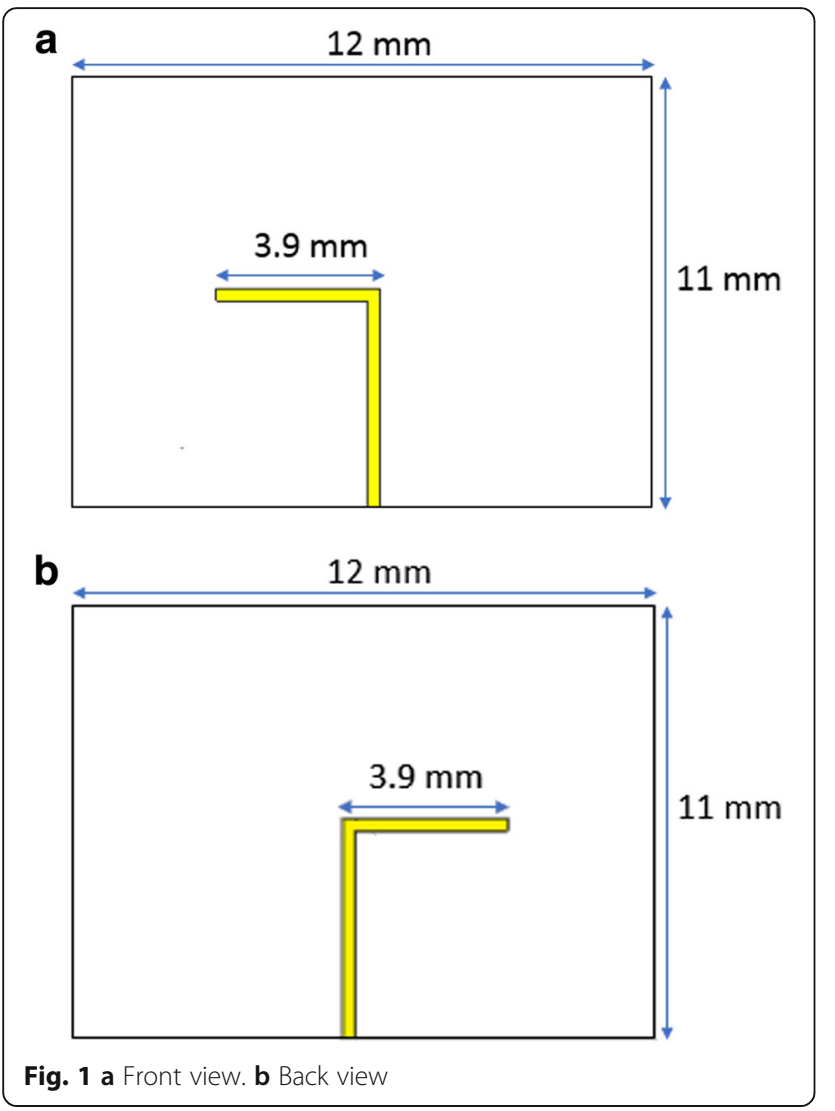

the high-gain characteristics. The novelty of the antenna that is presented in this paper is threefold: it offers the characteristics of [4-7], proposes a feeding structure to minimize the profile length and adds around $3 \mathrm{dBi}$ to the gain of a basic Yagi antenna, by incorporating a specific, variable refractive index-guided novel arrangement of arc-shaped parasitics, in front of the driver dipole. This launches the surface waves in such a way as to aid their transformation into directional far-field components. A prototype of the lowprofile antenna element which measures a square centimetre across has been fabricated and experimentally validated.

The rest of the paper is organized as follows: the antenna element design principle is introduced in Section 2, Section 3 discusses the antenna fabrication and experimental validation of the antenna element with the measurement results and Section 4 concludes.

\section{Antenna design principle}

The design philosophy adopted rests on the use and integration of three basic elements that form the antenna design: the driver dipoles, the printed reflector and the parasitics. The antenna was brought into operation over an evolved sequential design following first the principles. In the following, the design is discussed for each of the three constituent basic elements.

\subsection{Driver dipoles}

The substrate chosen for the printed antenna configuration has a dielectric constant $\varepsilon_{r}=3.55$, loss tangent $(\tan \delta)=0.0027$ and a thickness of $0.508 \mathrm{~mm}$. The substrate area was chosen to mitigate the surface waves and the fringing edge effects to a considerable extent by maintaining a peripheral distance between the structure to the boundaries greater than a quarter wavelength. The substrate area was thus set to $12 \mathrm{~mm} \times 11 \mathrm{~mm}$. A $\lambda / 2$ printed dipole centred at the chosen frequency of $14 \mathrm{GHz}$ was designed. The arms of the dipole were chosen to be printed

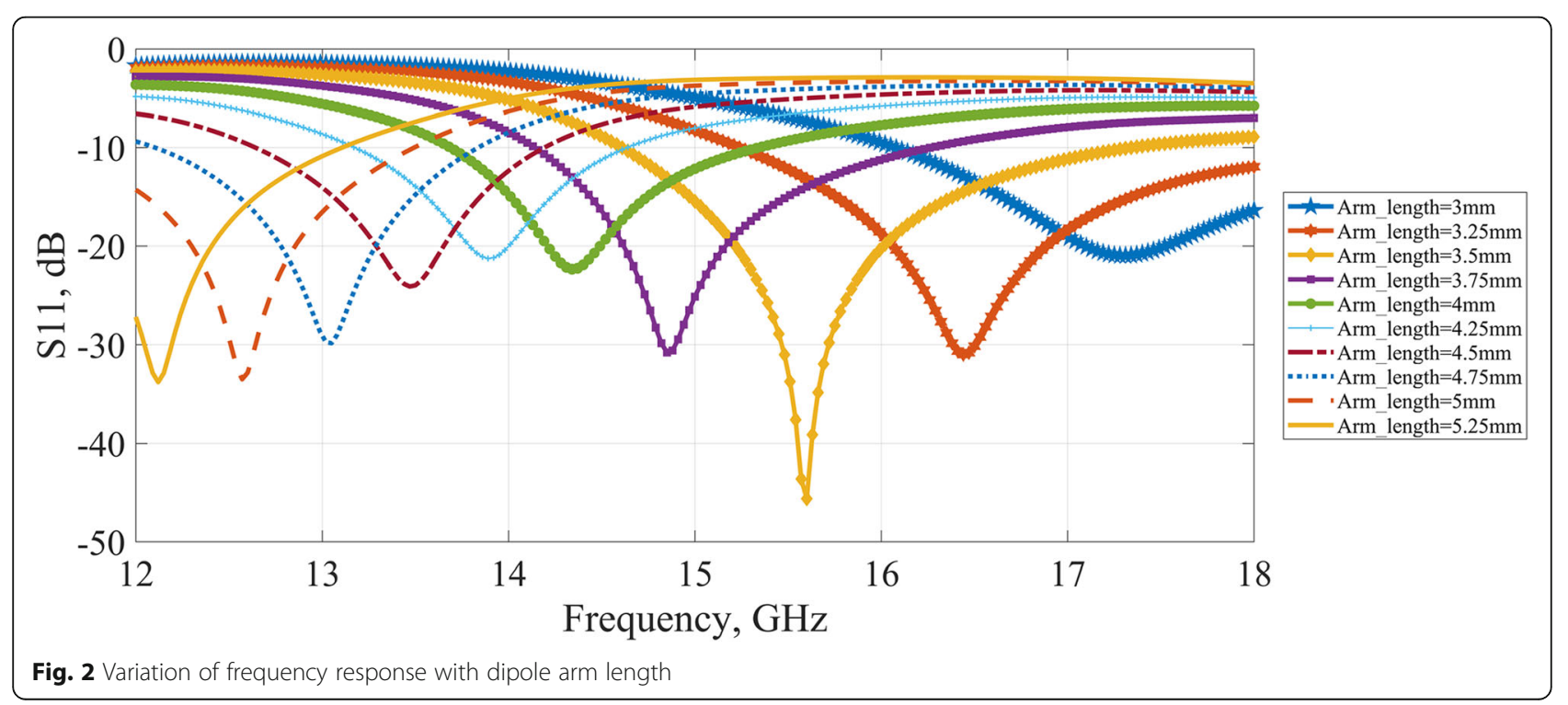




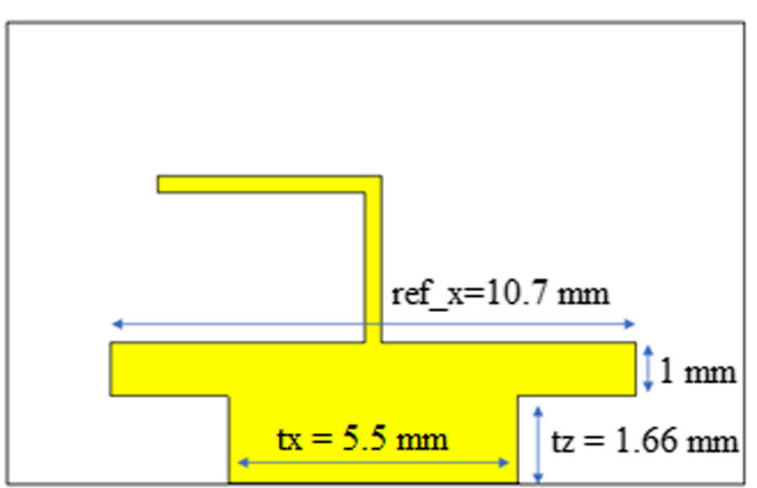

Fig. 3 Printed reflector profile

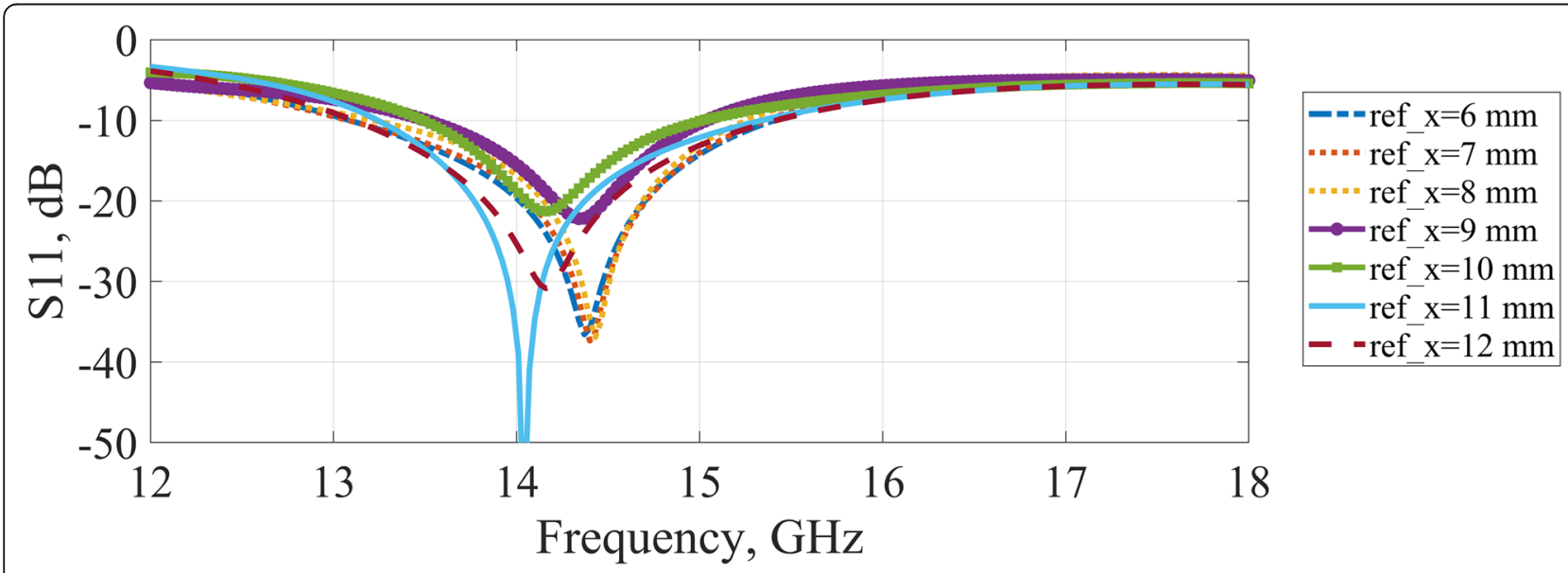

Fig. 4 Effect of the variation of ref_x on S11 frequency response

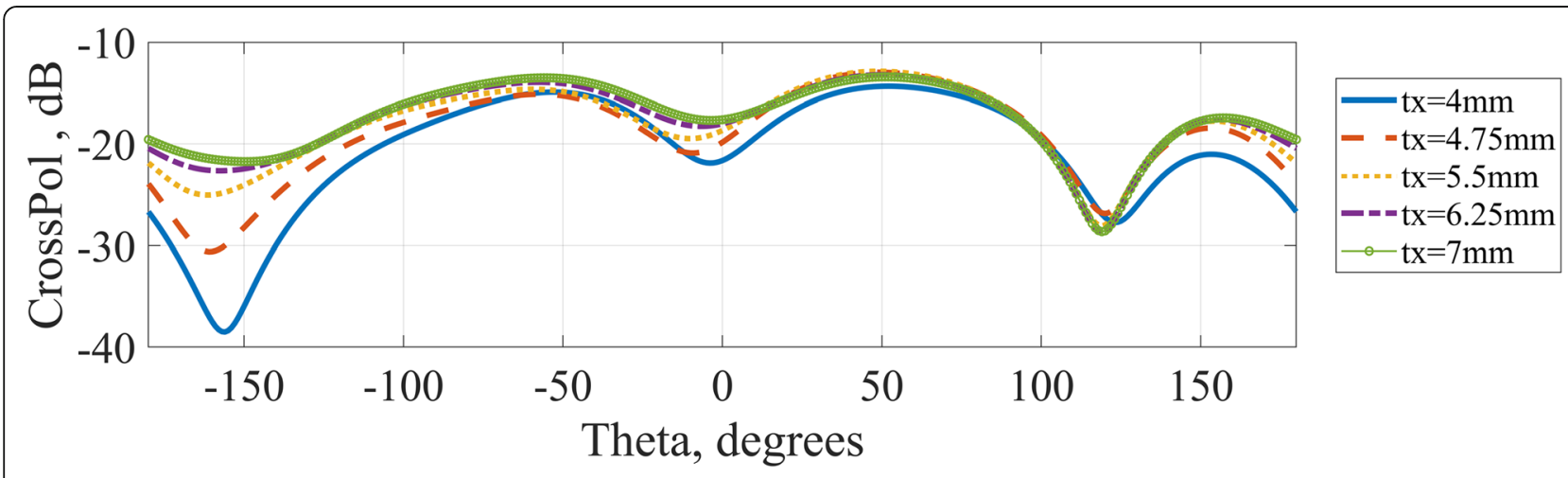

Fig. 5 Effect of the variation of tx (transition width) on cross-polar level for Phi0 plane 


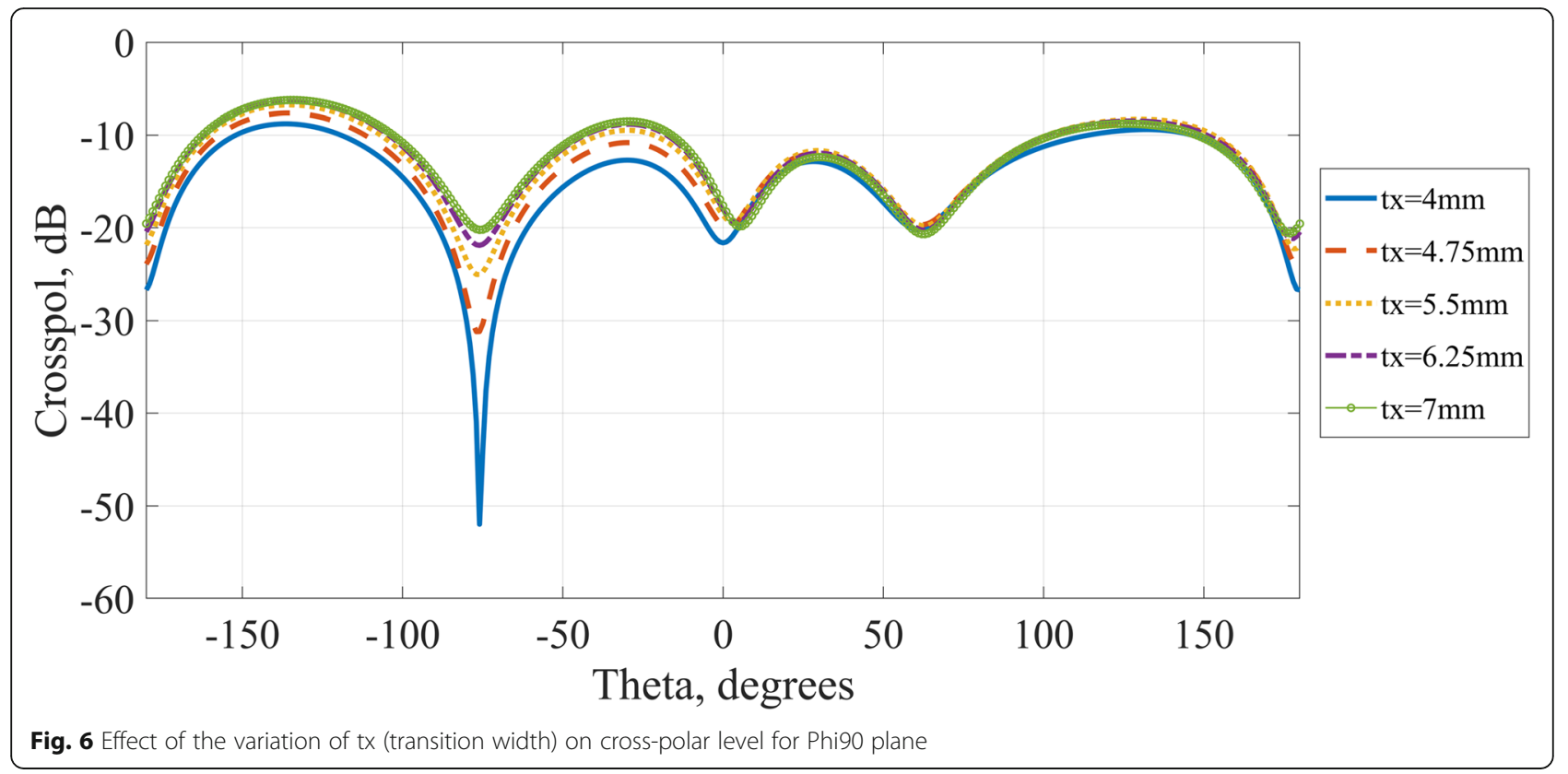

on opposite sides of the substrate to act as a selfbalancing structure. Figure 1a shows the front view of the design of an arm of the dipole on the substrate and Fig. 1b shows the back view of the other arm. Initially, the length of each arm was selected as $\lambda_{0} / 4$ at $14 \mathrm{GHz}$, equal to $5.35 \mathrm{~mm}$. The length of the arm was later fine-tuned through full-wave simulations to $3.9 \mathrm{~mm}$ to have the band centred at $14 \mathrm{GHz}$. A parametric variation of the arm length and its effect on S11 is shown in Fig. 2. For this parametric variation of other parameters such as the transition length and width, the reflector length and width are kept constant and only the arm length is varied in steps of $0.25 \mathrm{~mm}$, displaying the tuning behaviour over the $12-18 \mathrm{GHz}$ range for an arm length variation from $3 \mathrm{~mm}$ to $5.25 \mathrm{~mm}$. This tuning behaviour demonstrates the potential of the antenna to be reconfigured in frequency.

\subsection{The printed reflector}

The antenna design requires a printed reflector to enhance the directivity and the front-to-back ratio. The design of the printed reflector is performed to use the reflector both as a ground and as a supporting structure to the SMA feeder connector. This requirement makes the reflector a stepped design with the width (tx) of the section facing the SMA near to $\lambda_{0} / 4$ at $14 \mathrm{GHz}$ and equal to $5.5 \mathrm{~mm}$. The actual

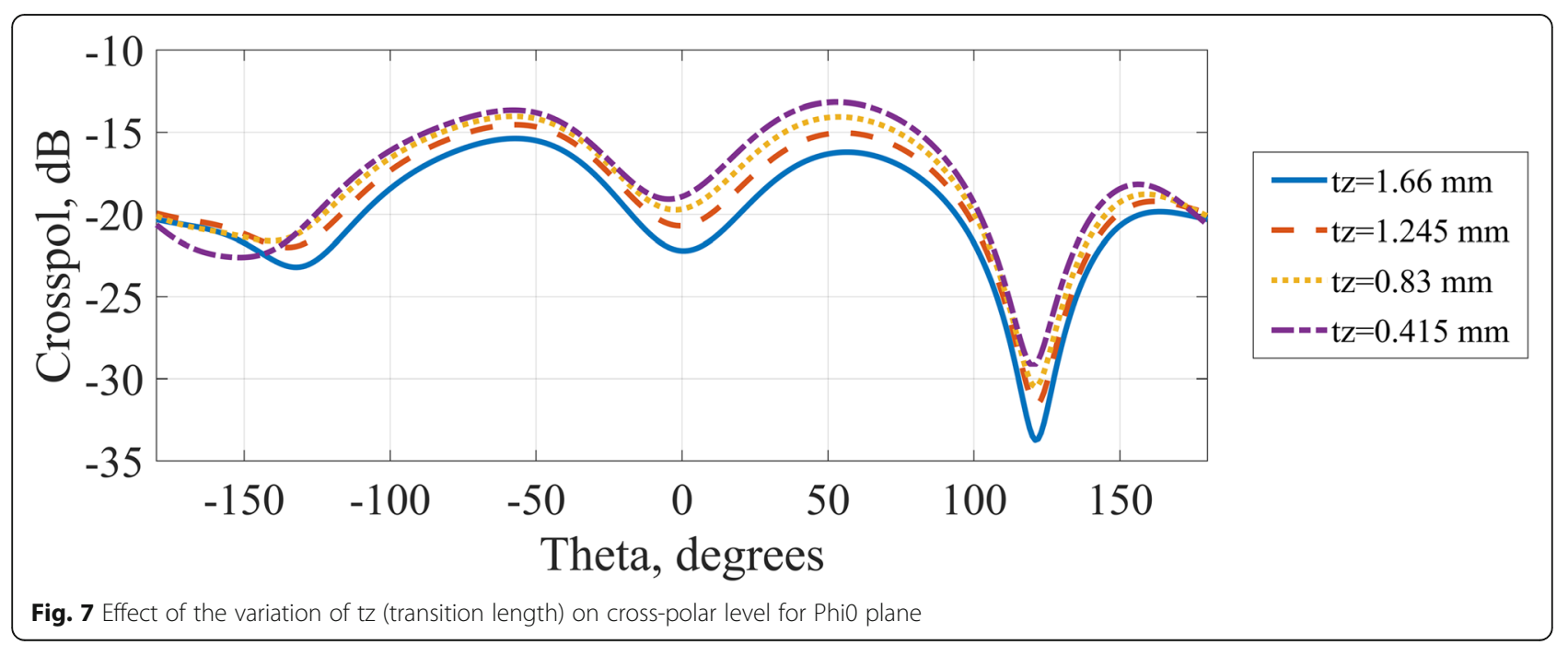




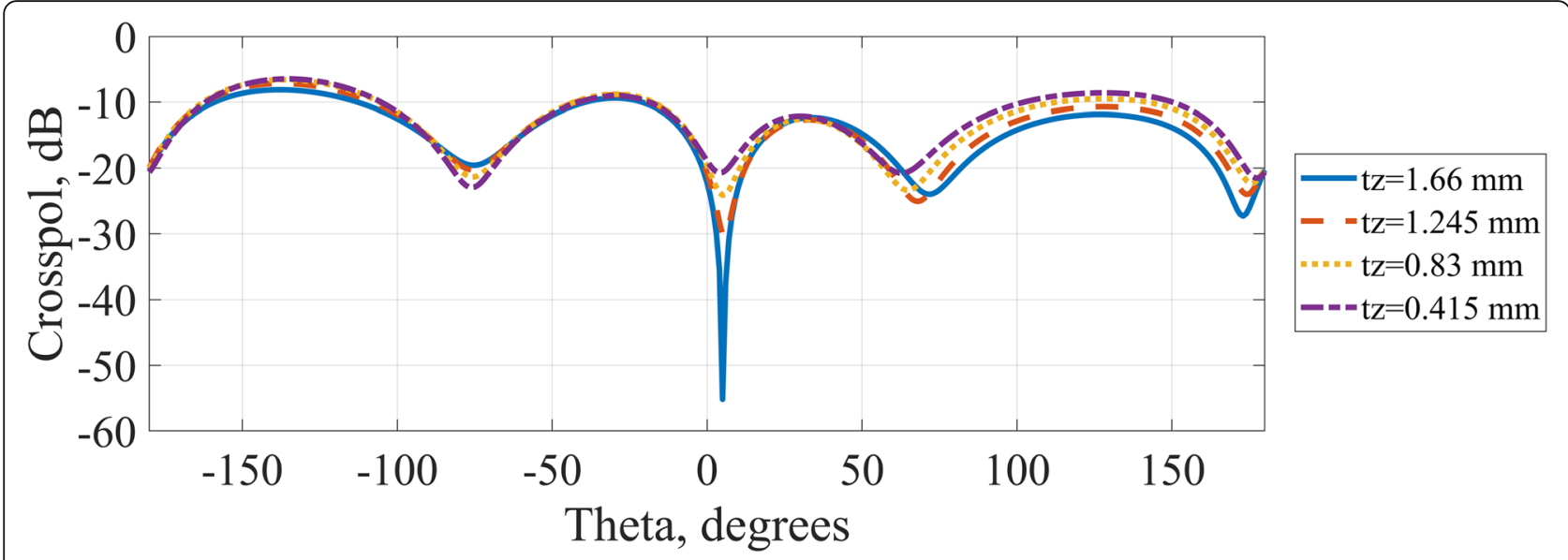

Fig. 8 Effect of the variation of tz (transition length) on cross-polar level for Phi90 plane

reflector length (ref_x) is $\lambda_{0} / 2$ at $14 \mathrm{GHz}$ equal to $10.7 \mathrm{~mm}$. The final printed reflector, which is printed on the face opposite to the side where the SMA pin connects, is shown in Fig. 3 with its optimized dimensions.

A parametric study was performed over the transition width (tx), transition length (tz) and the reflector length (ref_x). As shown in Fig. 4, the reflector length variation (ref_x) does not affect the frequency behaviour excessively. This strengthens the dependence of the frequency behaviour of the antenna mainly on the arm length as shown in Fig. 2 with the reflector aiding in enhancing the front-toback ratio. A redesign of the reflector is needed for each tuning frequency. It was observed that the transition dimensions affect the amount of realizable cross-polar levels. A parametric study of this dependence was performed by varying the transition length (tz) and transition width (tx) vs the observable cross-polar levels at Phi0 and Phi90 principal cut planes, and results are shown in Figs. 5, 6, 7 and 8. It was seen that the effect of varying the transition width (tx) is not as pronounced as the effect of varying the transition length (tz). Variation of $\mathrm{tz}$ controls the observable cross-polar levels. As seen from Fig. 8, a substantial increase in the cross-polar levels is observed around boresight for variations over tz. The length before the transition step is a crucial factor that controls the cross-polar levels of the antenna and therefore has been deduced to be equal to $1.66 \mathrm{~mm}$ both by considering the surface current distribution as shown in Fig. 9 and the cross-polar levels of the pattern. Here the surface current distribution is stronger on the dipole arms for the depicted cases of absolute surface current phase of $0^{\circ}, 90^{\circ}$ and $180^{\circ}$, showing that the reflector performs the field enhancement through reflection.
The full-wave solver calculated values of the crosspolar levels after the full design of the antenna helps also in deciding the transition step length of $\mathrm{tz}=$ $1.66 \mathrm{~mm}$ and plays a critical role in the design.

\subsection{The parasitics}

In Fig. 9, the surface current distribution shows the currents strongly distributed on the dipole arms. It is intended to couple the electric field due to this onto the parasitics in a constructive way to enhance the far-field gain. To achieve this, a systematic distribution of parasitics is proposed. The first parasitic that couples the dipole arm field is a rectangleshaped parasitic as shown in Fig. 10. The electric field coupled onto this rectangle-shaped parasitic is distributed along two oblique directions, using two symmetrically placed square parasitic elements, whose dimensions are $0.05 \lambda \times 0.05 \lambda$ that translates to $1 \mathrm{~mm} \times 1 \mathrm{~mm}$, each suitably spaced from each other to enhance coupling, while equally distributing the electric field along two symmetric oblique directions. Figure 10 shows the rectangle-shaped parasitic and the two square parasitics coupling onto the dipole. The capacitive coupling gap between them was set to maximize the coupling.

In the following, the design of the arc-shaped parasitics is discussed. The shape of the parasitics was chosen as an arc, to channel the electric field obliquely. In addition, it also maximizes the coupling by maximizing the amount of exposed surface area that couples onto the next parasitic. The fibre-optic principle was used to position the parasitics along the antenna plane in front of the square resonators. The design derives its inspiration by analogy to a graded-index fibre-optic cable, whose core can be 
considered analogous to a certain cuboidal volume region, lying directly below the endfire central axis of the antenna, where the graded real refractive index is set to a maximum. The outward displacement towards the edges can be considered to represent the cladding-where there is a gradient decrease of the real refractive index moving away from the centre. This is achieved by the methodical placement of the arc-shaped parasitics in front of the driver dipoles over the antenna plane. The procedure to arrive at this is discussed next.

The dielectric substrate used in the antenna can be considered a homogeneous slab for the frequencies under consideration. The refractive index is the reconfigurable parameter that is used to direct the mechanism of radiation. Then according to [9], the general procedure for the retrieval of the material parameters, in this case, the real part of the refractive index is as follows: the arc-shaped parasitics are placed so that they focus the electric field towards the boresight axis. A one-dimensional transfer matrix $T$ relates the fields on one side of a slab to the other. For a homogeneous 1D slab, it takes the form (1):

$$
T=\left(\begin{array}{cc}
\cos (n k d) & -\frac{z}{k} \sin (n k d) \\
\frac{k}{z} \sin (n k d) & \cos (n k d)
\end{array}\right)
$$

where $n$ is the refractive index, $z$ is the wave impedance, $k$ is the wave number and $d$ is the thickness of the slab. A scattering matrix relates the impinging field amplitude to the outgoing field amplitude. The elements of the $S$ matrix can be related to the elements of the $T$ matrix as in (2) [10]:

$$
\begin{aligned}
& S_{21}=\frac{2}{T_{11}+T_{22}+\left(i k T_{12}+\frac{T_{21}}{i k}\right)}, \\
& S_{11}=\frac{T_{11}-T_{22}+\left(i k T_{12}-\frac{T_{21}}{i k}\right)}{T_{11}+T_{22}+\left(i k T_{12}+\frac{T_{21}}{i k}\right)}, \\
& S_{22}=\frac{T_{22}-T_{11}+\left(i k T_{12}-\frac{T_{21}}{i k}\right)}{T_{11}+T_{22}+\left(i k T_{12}+\frac{T_{21}}{i k}\right)}, \\
& S_{12}=\frac{2 \operatorname{det}(T)}{T_{11}+T_{22}+\left(i k T_{12}+\frac{T_{21}}{i k}\right)}
\end{aligned}
$$

For a homogeneous slab from equation (1),

$$
T_{11}=T_{22}=T_{S} \text { and } \operatorname{det}(T)=1
$$

And the $S$ matrix is symmetric. Using the information in equation (1), (3) and (4) can be derived:

$$
\begin{aligned}
& S_{21}=S_{12}=\frac{1}{\cos (n k d)-\frac{i}{2}\left(z+\frac{1}{z}\right) \sin (n k d)} \\
& S_{11}=S_{22}=\frac{i}{2}\left(\frac{1}{z}-z\right) \sin (n k d)
\end{aligned}
$$

Solving equations (3) and (4) for $n$ and $z$ yields (5) and (6) [9]:

$$
\begin{aligned}
& n=\frac{1}{k d} \cos ^{-1}\left[\frac{1}{2 S_{21}}\left(1-S_{11}^{2}+S_{21}^{2}\right)\right] \\
& z=\sqrt{\frac{\left(1+S_{11}\right)^{2}-S_{21}^{2}}{\left(1-S_{11}\right)^{2}-S_{21}^{2}}}
\end{aligned}
$$

The design principle of the antenna revolves mainly around equation (5) which gives the expression for the refractive index as a function of the reflection and transmission $S$ parameters and the slab length $d$. The application of equation (5) is first performed over a single-unit cell of the arc-shaped parasitics (ASP) as shown in Fig. 11. The unit cell is excited by waveguide ports excited on the two faces of the dielectric slab on which the ASP is printed. On running the simulation in a full-wave solver for the desired frequency of interest, the $S_{11}$ and $S_{21}$ values can be obtained. The length of the arc of the ASP is $1.7 \mathrm{~mm}$, around $\lambda / 10$ at $14 \mathrm{GHz}$.

Of the total 10 ASPs selected to enhance the endfire gain, five are on the left side of the endfire axis and the other five are symmetrically on to the other side. Equation (5) is used to calculate the refractive index as illustrated in Fig. 12 which depicts the way the refractive indices were calculated starting from the endfire central axis, towards the extreme left. Since the structure is symmetrical across the endfire axis, the same calculation of the refractive index holds for the right side as well.

From Fig. 12, starting from the extreme right, i.e. the central ASP, setting up the $S$ parameter extraction and retrieval of the refractive index on the full-wave solver returns values specific to the band of interest and length of the slab $(d)$ equal to $1 \mathrm{~mm}$. This procedure is repeated for other four cases of $d=1.5,2$, 2.5 and $3 \mathrm{~mm}$. The distance along the $x$-axis in Fig. 12 is not to scale, it is a pictorial depiction for clarity purposes of describing the setup. This methodology extracts the real part of the refractive index for this kind of unit cells in a sequential way. Their values as a function of frequency are plotted in Fig. 13, where the real part of the refractive index variation on the 

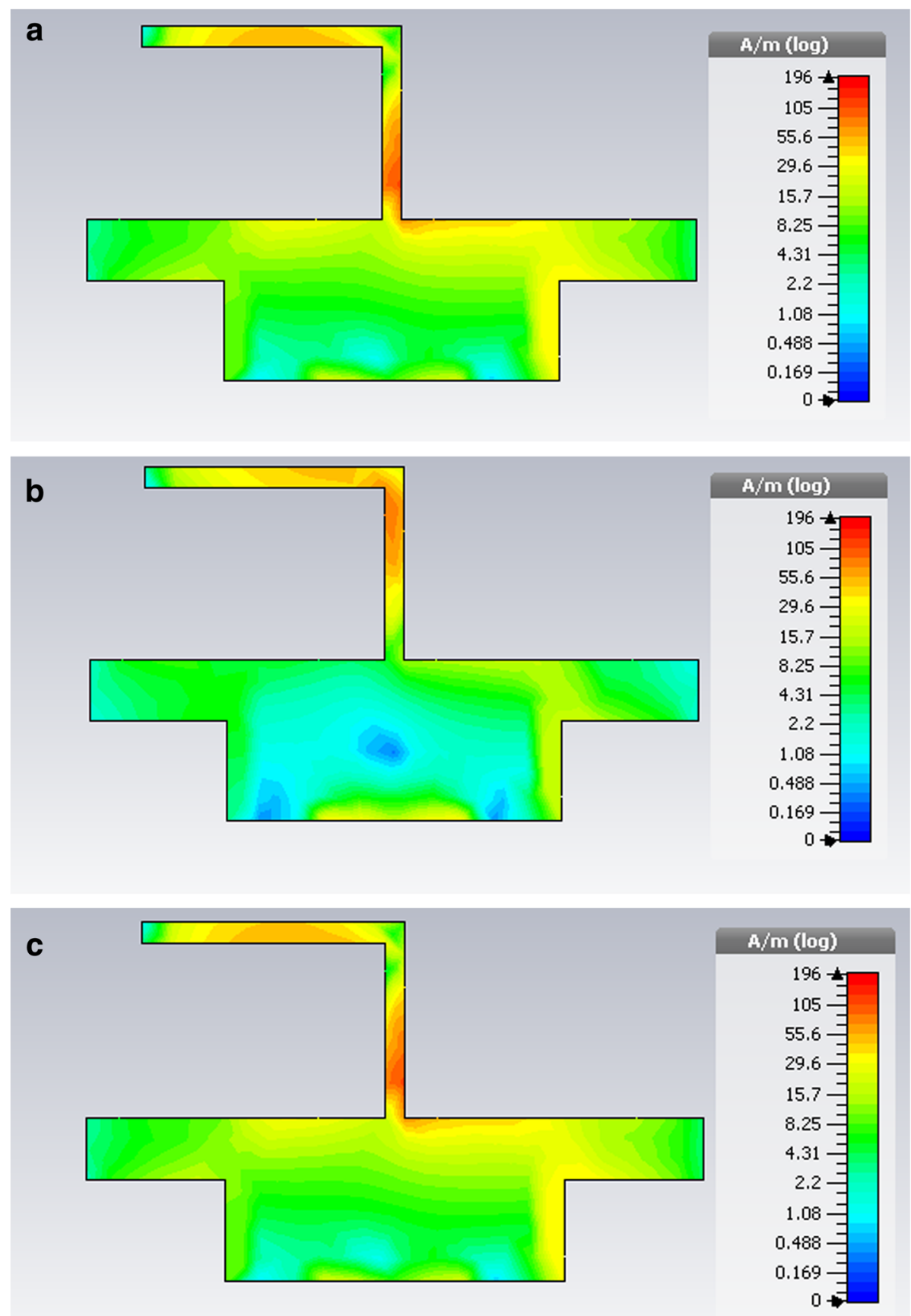

Fig. 9 Surface current distribution on the reflector at phase a $0^{\circ}, \mathbf{b} 90^{\circ}$ and $\mathbf{c} 180^{\circ}$ at $14 \mathrm{GHz}$

structure is shown, with different curves signifyingthe distance between the arc-shaped parasitic element and the square resonator (d) $-1 \mathrm{~mm}$ being the closest one near to the central axis and $3 \mathrm{~mm}$ for the one on the extreme flaring edges. The imaginary part of the refractive index in these cases is near zero signifying minimum loss in radiating energies.
Figure 14 shows gradient depiction of the variation of the refractive index from the centre towards the edges with dark blue representing the highest real part of refractive index and lighter shade depicting a decreased value. This distribution of the real part of the refractive index makes the antenna behave analogous to a graded-index optical fibre that focuses 


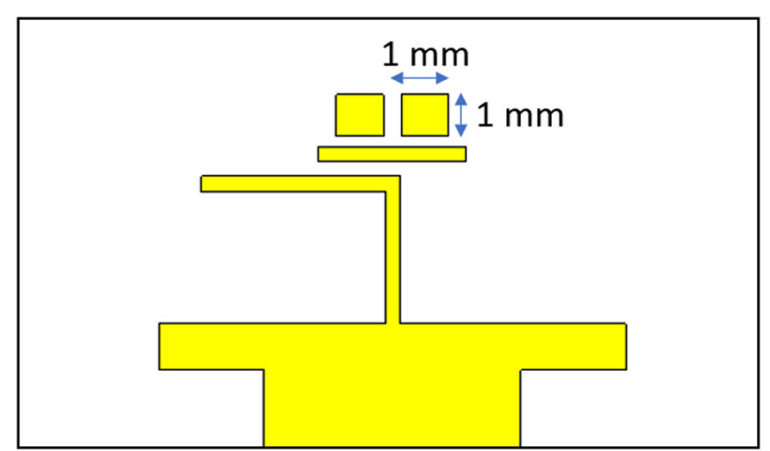

Fig. 10 Rectangle-shaped parasitic and the two square parasitics coupling onto the dipole

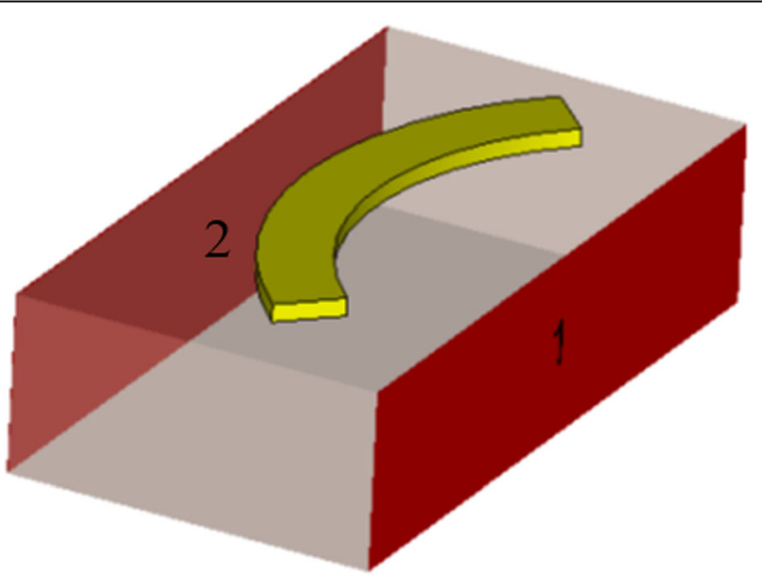

Fig. 11 A single-unit cell of the arc-shaped parasitic

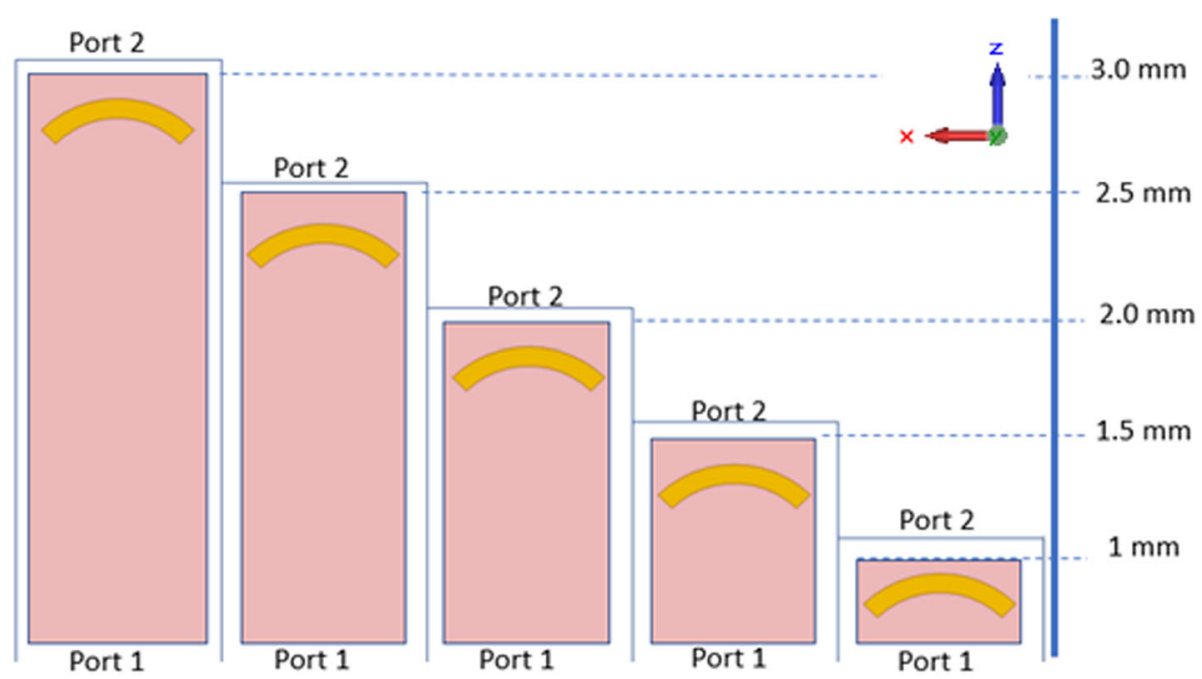

Fig. 12 Illustration depicting the way of refractive index retrieval 


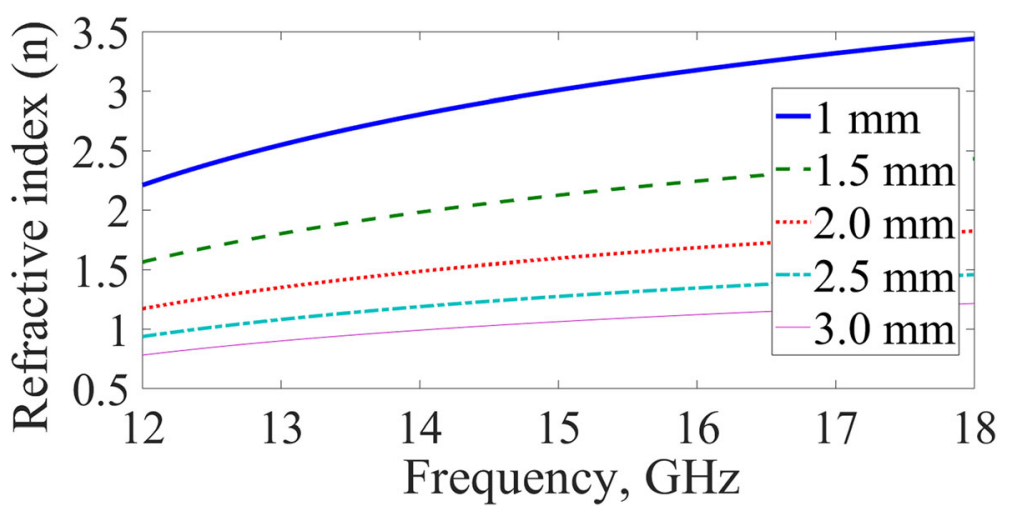

Fig. 13 Real part of the refractive index of the unit cell parasitic arc-shaped resonators from centre towards edges

energy towards the centre increasing the gain of the antenna. The simulation results of the antenna have been presented in [11].

\subsection{Effect of antenna backplate}

The antenna design described above in Sections 2.1, 2.2 and 2.3 is fed with a suitably designed $50-\mathrm{ohm}$ coaxial SMA connector as in Fig. 15a. As the goal is to use the antenna in an array, a common mechanical support plate to connect and support multiple antenna elements is needed. The supporting plate is designed with a square hole in the centre as shown in Fig. 15b. The presence of the supporting plate with a hole has two functions: (1) It sufficiently isolates the ground of the designed antenna and maintains a purity in the tuning of the resonant frequency. (2) It enhances the obtained gain pattern by providing for improved cross polar levels. The 3D co-polar gain radiation pattern obtained out of

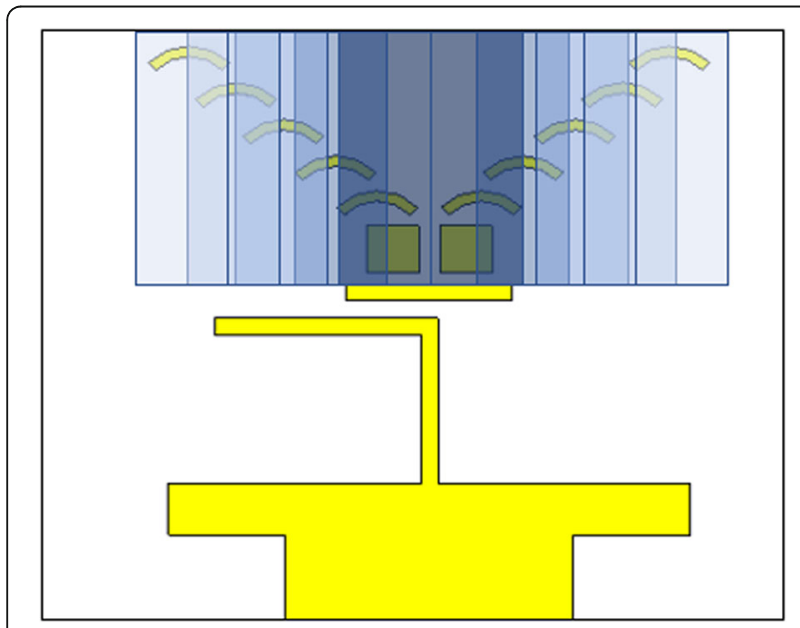

Fig. 14 Gradient depiction of the gradient variation of the refractive index the antenna without the backplate and with the holed backplate is shown in Fig. 16. The 3D cross-polar gain pattern for the same two cases is shown in Fig. 17.

The cross-polar discrimination (XPD) improvement for the case with the backplate is compared with the case without and is shown in the Cartesian plot in Fig. 18. It can be clearly seen that the XPD is improved considerably with the use of a holed

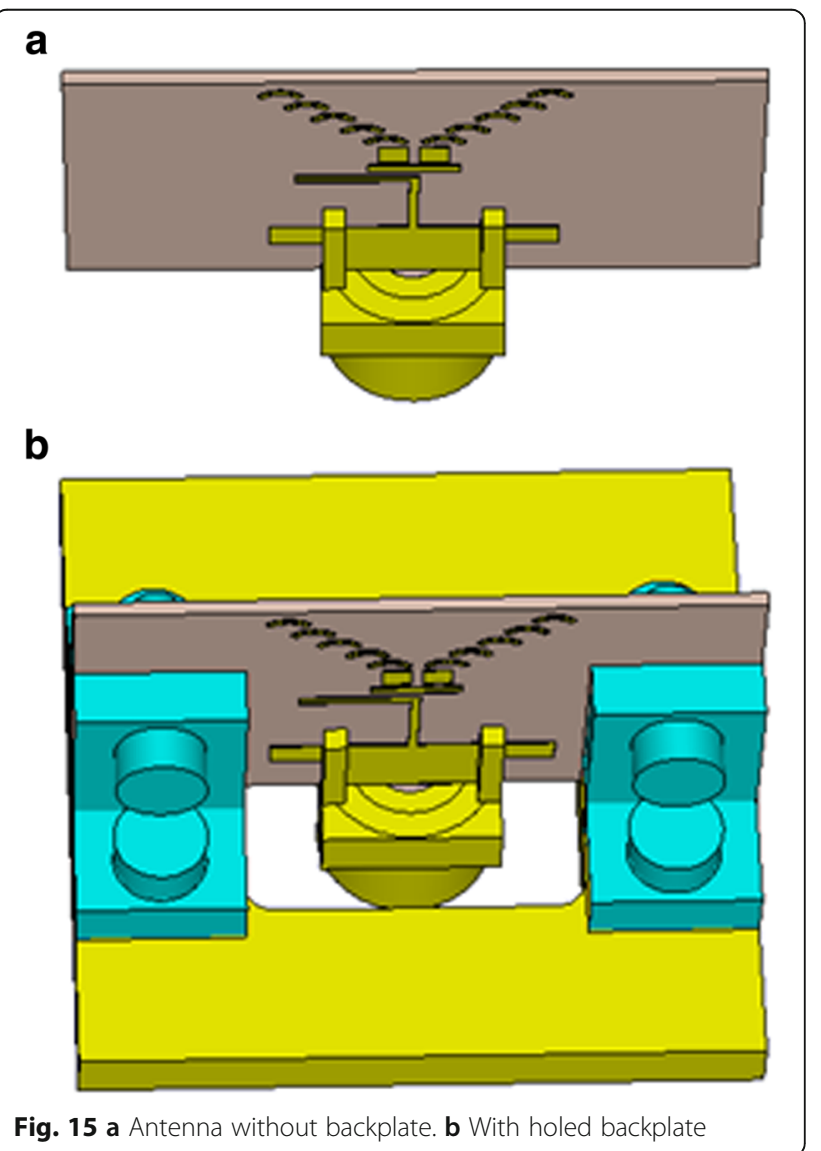



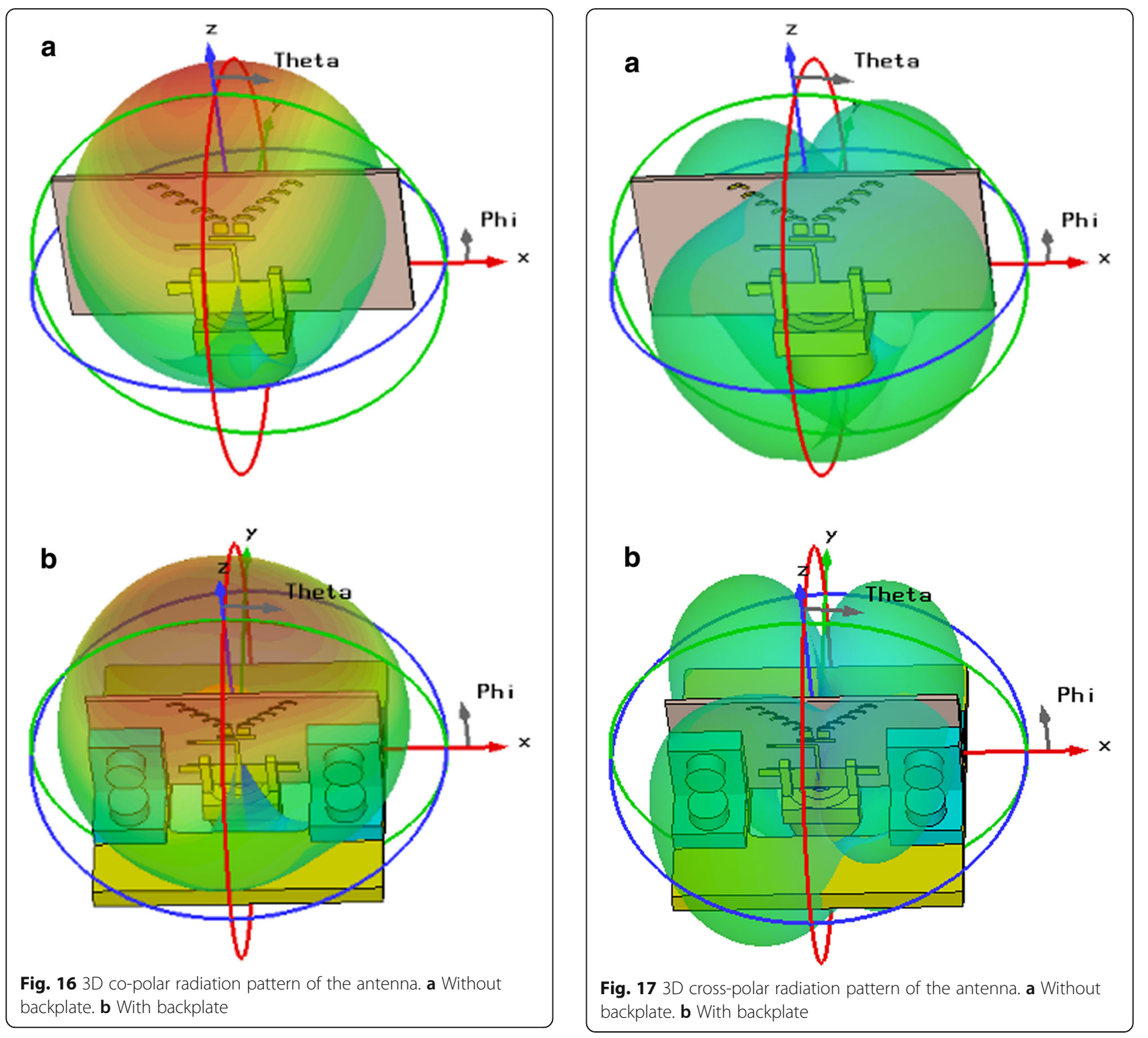

Fig. 16 3D co-polar radiation pattern of the antenna. a Without backplate. $\mathbf{b}$ With backplate

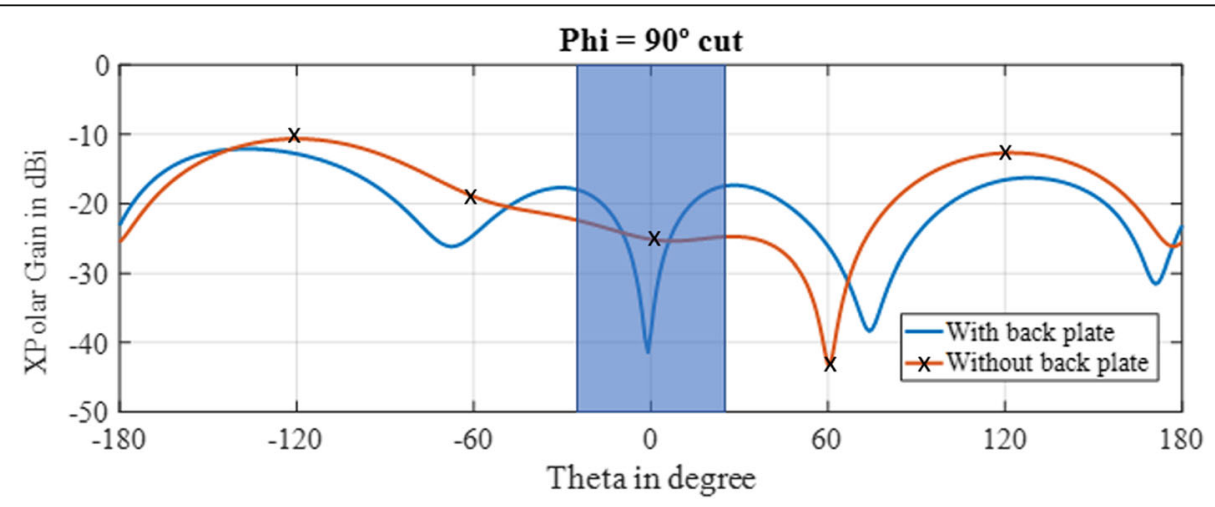

Fig. 18 XPD cross polar discrimination comparison for cases of without and with back plate 


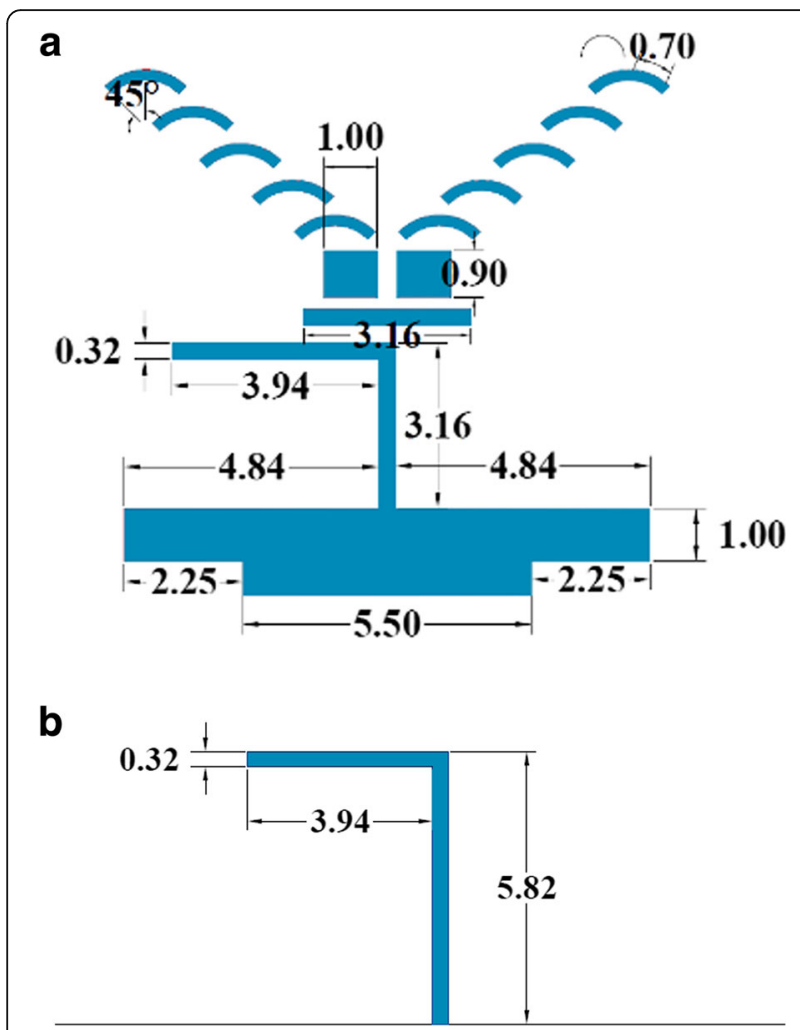

Fig. 19 Low-profile, high-gain quasi-Yagi antenna element dimensions. a Front view. b Back view

backplate by $15 \mathrm{~dB}$ along the boresight direction for a beamwidth of $20^{\circ}$.

\section{Measurements and discussion}

The designed antenna obtained by combining the steps outlined in Section 2 is shown in Fig. 19 and consists of a dipole driver element with arms printed

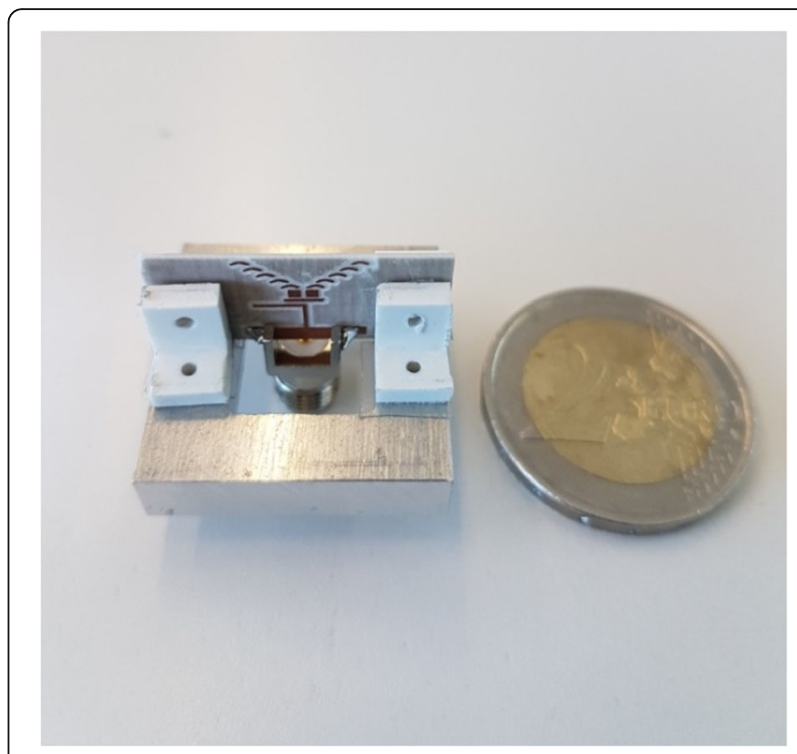

Fig. 21 Fabricated prototype with size of a square centimetre and the supporting structure

on opposite sides of the substrate and a printed reflector to improve the forward radiation. A parasitic rectangular director couples the E-field into the two square parasitic elements that act as driving sources to the arrangement of the arc-shaped parasitic unit cell. These are flared in a V shape and spaced $0.2 \mathrm{~mm}$ from each other. The dimensions of the proposed quasi-Yagi antenna are shown in Fig. 19. The simulations were carried out in Ansoft HFSS. The antenna has an operational band from 13.5 to $15 \mathrm{GHz}$, with a fractional bandwidth of $10.52 \%$. The energychannelling behaviour of the arc-shaped transitions is illustrated by the simulated current densities shown in Fig. 20 at $14 \mathrm{GHz}$. To check the validity of the

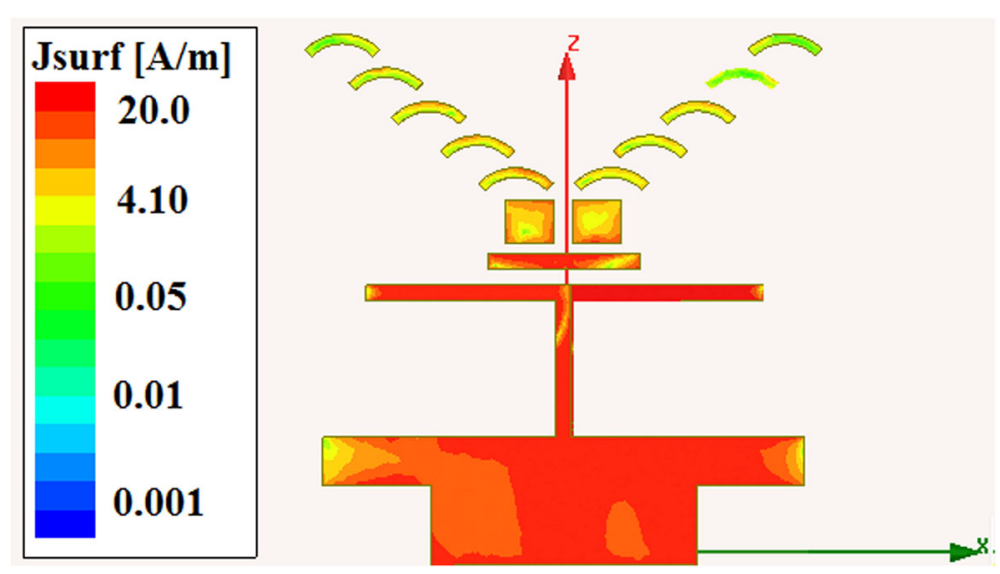

Fig. 20 Surface current distribution at $14 \mathrm{GHz}$ 


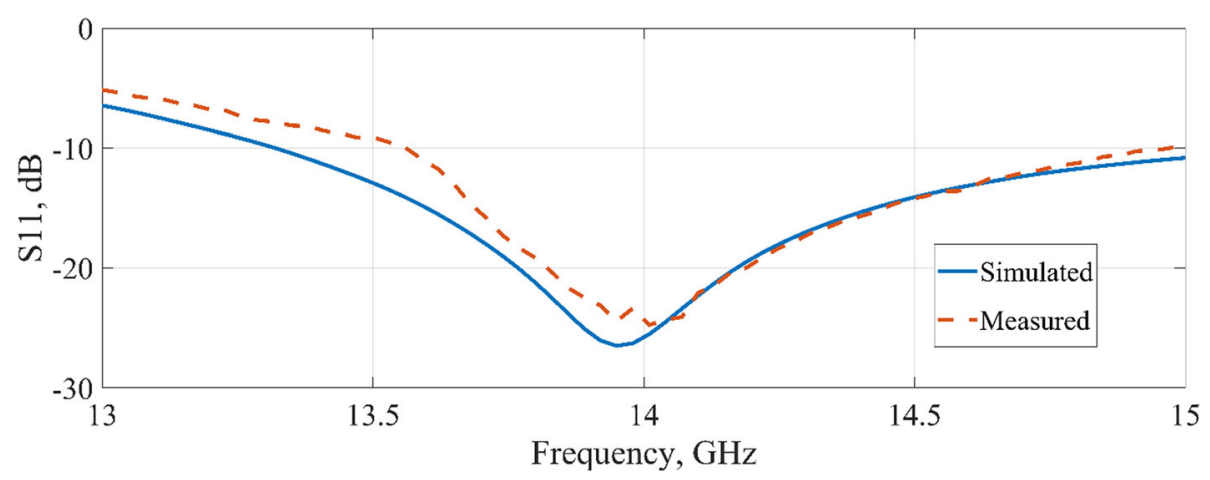

Fig. 22 Simulated and measured $S_{11}$ behaviour of the antenna element operating from $13.5 \mathrm{GHz}$ to $15 \mathrm{GHz}$ for 10-dB return loss

proposed structure, a prototype was fabricated as shown in Fig. 21 and measured in an anechoic chamber. The simulated and measured $S_{11}$ are compared respectively by a blue line and red dashed lines in Fig. 22: a 10-dB return loss bandwidth of approximately $1.5 \mathrm{GHz}$ around $14 \mathrm{GHz}$ is obtained. The printed antenna was backed by an aluminium supporting structure, with a square gap in the middle to allow for the SMA feeding and to enhance the XPD.

Besides, both the principal pattern cuts as well as boresight direction $\left(0^{\circ}\right)$ gain were measured in a farfield anechoic chamber, directly attaching the prototype to the support plate of the roll over azimuth positioner, whose dimensions are much larger than the base of the fabricated prototype of Fig. 21. The model of the support plate is shown in Fig. 23 to give a comparison of the size. The simulated (blue dashed lines) and measured (red lines) radiation patterns for the co-polar component are compared in Figs. 24 and 25 for both principal planes, elevation and azimuth cuts, respectively. A slight asymmetry of the current distributions can be observed that explains the approximately $20^{\circ}$ deviation of the maximum on the elevation cut shown by the red line on Fig. 24 a. An almost symmetric response is obtained in the azimuth cut shown in Fig. 24b. The large size of the supporting plate generates a reflected signal that adds to the prototype radiation causing an increased degradation of the measured patterns when departing from boresight $\left(0^{\circ}\right)$. Also, both the roll positioner and mast covered by absorbers block the measured signal on the back side which translates to

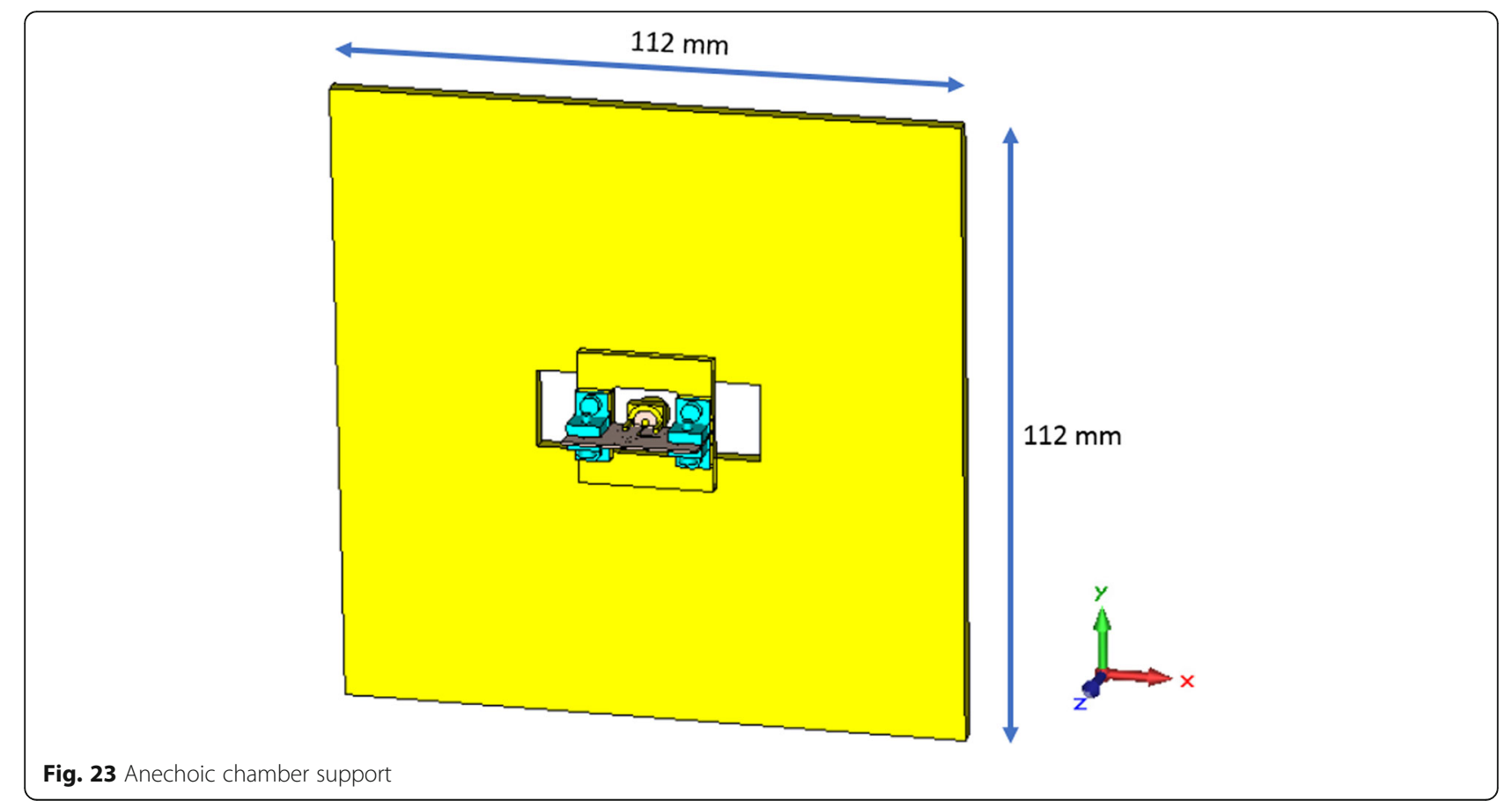



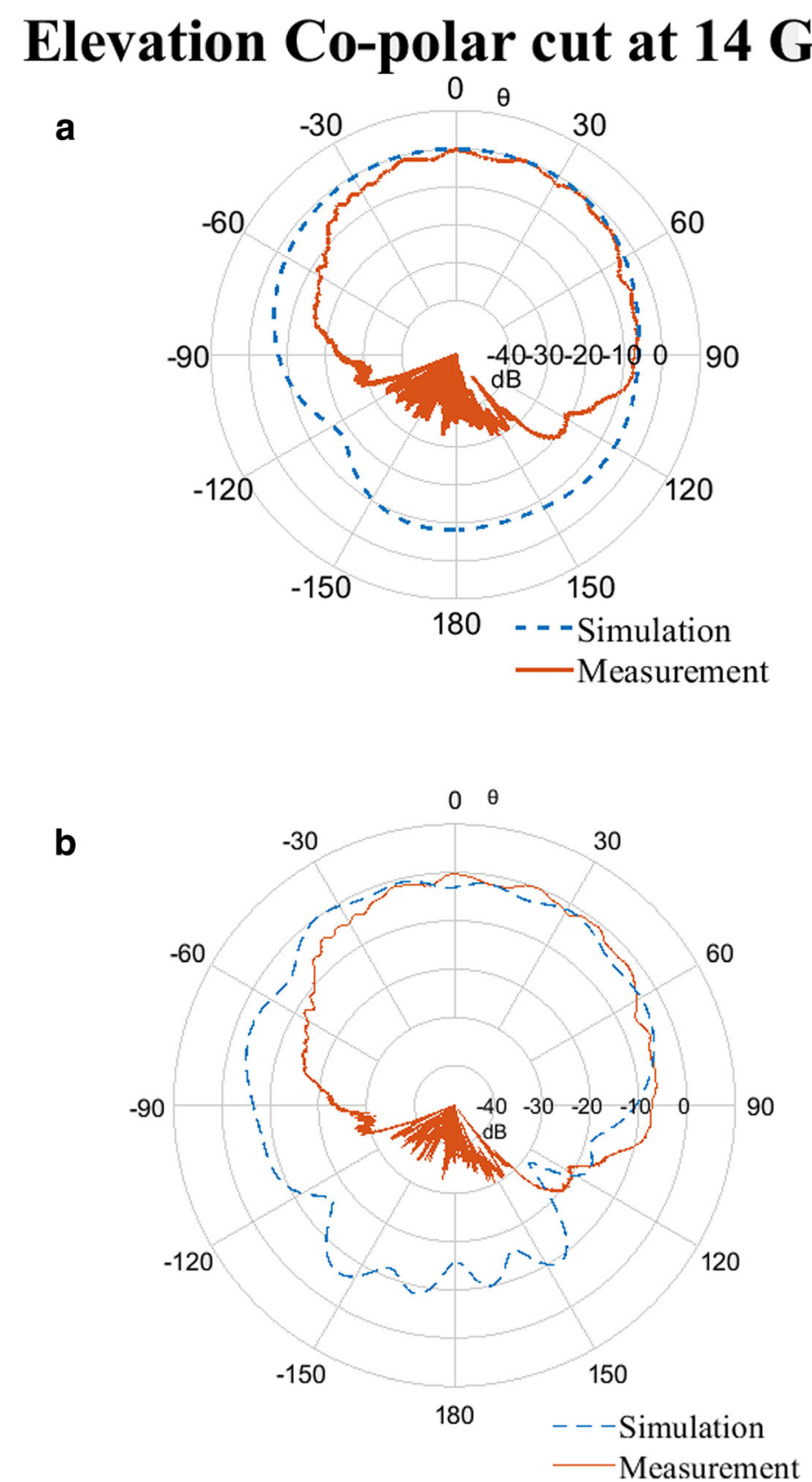

Fig. 24 Comparison of simulated and measured elevation cut. a Simulation curve is without anechoic chamber backplate. b Simulation curve is with anechoic chamber backplate

minimum back lobes in the measured pattern as in Fig. 24a and b. A reasonable agreement between measurements and simulation patterns is obtained.

Moreover, the gain of the antenna was measured using the two-antenna method for its variation over frequency. The simulated (blue dashed line) and measured (red line) gains in the boresight direction are compared in Fig. 26. A gain variation of $1 \mathrm{~dB}$ is seen in the band of interest that can be attributed to the ripple associated with the variations of the direct and reflected signal with frequency. The measured gain at $14 \mathrm{GHz}$ was around 6.0 $\mathrm{dBi}$ and keeps around $0.5 \mathrm{~dB}$ below the theoretical one in the 10-dB return loss bandwidth. Based on these results and preliminary theoretical checks of some modifications of the structure, oriented to symmetrize the current distributions on it, the proposed quasi-opticsinspired design methodology for low-profile endfire antenna can be considered experimentally validated. 

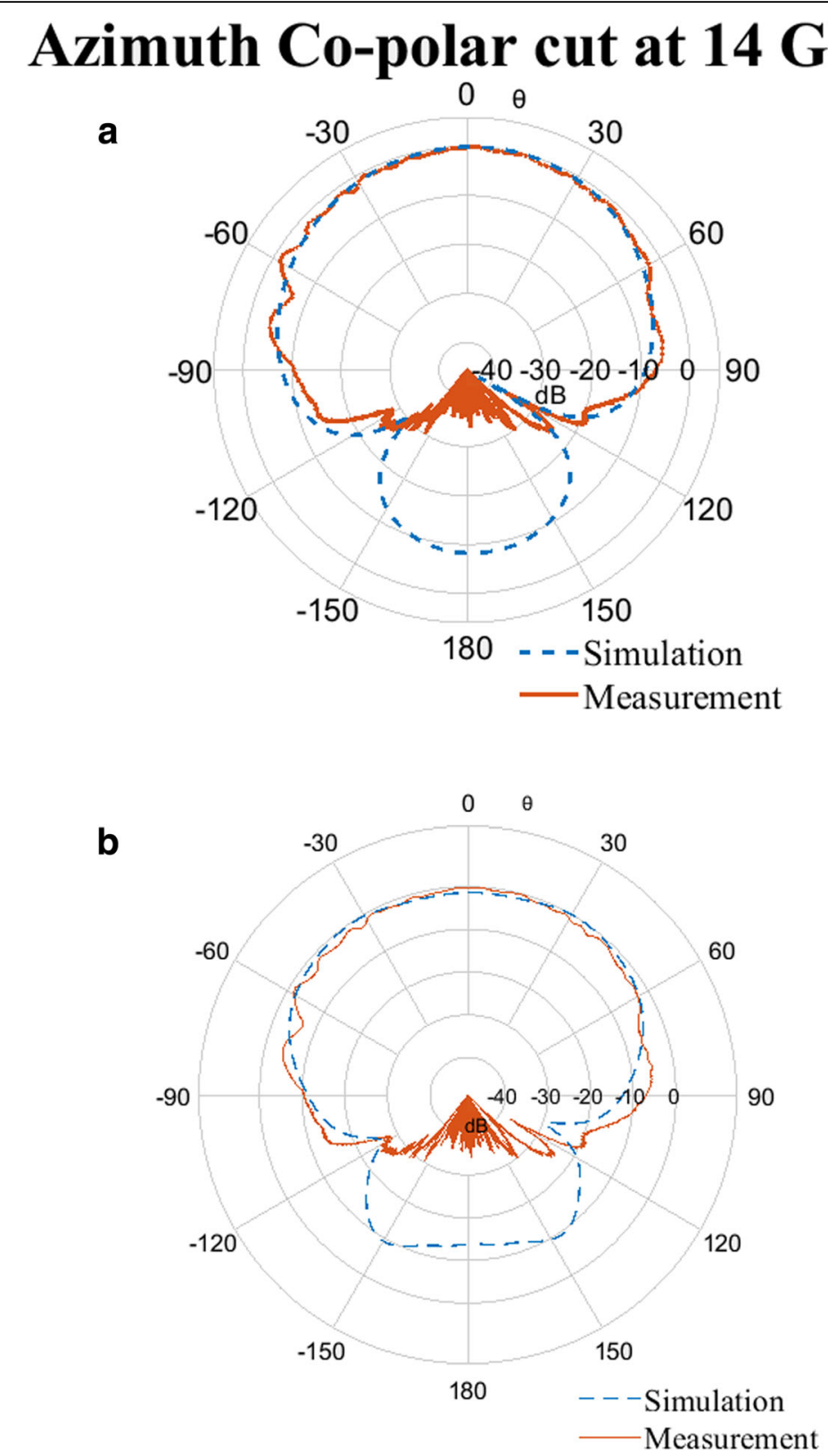

Fig. 25 Comparison of simulated and measured azimuth cut. a Simulation curve is without anechoic chamber backplate. b Simulation curve is with anechoic chamber backplate

\section{Conclusion}

A low-profile endfire antenna element inspired on fibre-optic principles is presented. A gradientrefractive index-based theoretical framework of the design, supported by parametric variation, is discussed. The antenna prototype has been fabricated and experimentally validated. It has an operation over $1.5 \mathrm{GHz}$ of bandwidth centred around $14 \mathrm{GHz}$ with a directional high-gain radiation pattern. The inherent layered structure when incorporated in an array aids to the better cooling of the antenna front end. The antenna element can find potential use in multi-user massive MIMO antenna arrays as well as in multibeam switching antenna arrays for 5G. The prototype has been adopted in a massive MIMO array and its performance has been validated. These findings are presented in [12] dedicated to the design and implementation of the massive MIMO array and its multibeam characteristics. Moreover, a performance evaluation of the fabricated antenna in a unique 


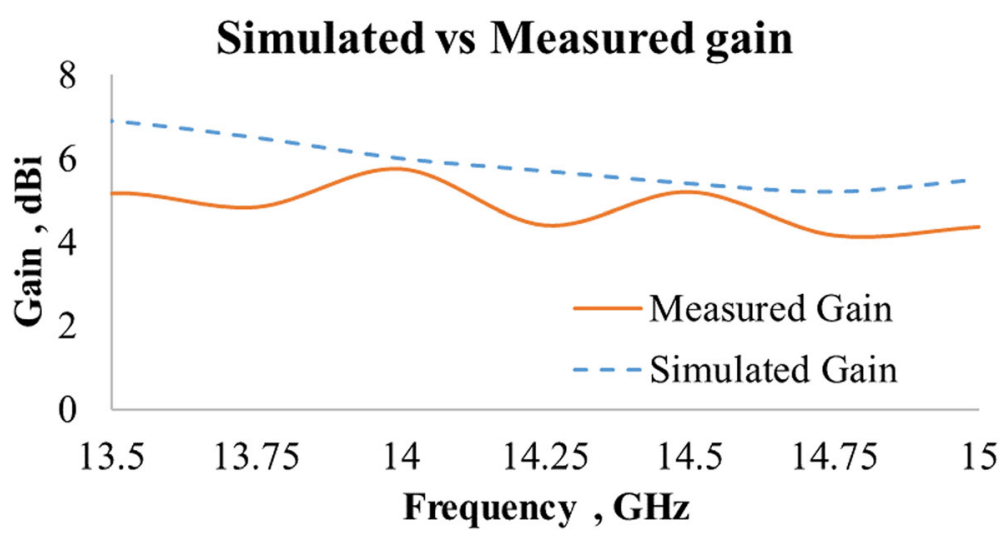

Fig. 26 Simulated and measured gain plot vs frequency

reconfigurable array setup applied to practical scenarios using spatial modulation is presented in [13].

\section{Abbreviations}

5G: 5th generation; ASP: Arc-shaped parasitic; CPS: Coplanar stripline; F/ B: Front-to-back ratio; HFSS: High-frequency structure simulator; MIMO: Multiple-input multiple-output; SMA: SubMiniature version A; XPD: Cross-polar discrimination

\section{Authors' contribution}

VB proposed the main idea, completed the experiments, analysed the results and wrote the paper. BB contributed in the pattern measurements. LC and $\mathrm{JB}$ read the paper and contributed critical constructive reviews. All authors read and approved the final manuscript.

\section{Funding}

This work was supported by the $5 \mathrm{G}$ wireless project that has received funding from the European Union's Horizon 2020 research and innovation programme under grant agreement No. 641985.

\section{Availability of data and materials}

The data used in the analysis and validation of the proposed antenna was generated by measurements at the vector network analyser and the anechoic chamber. Though this data has been presented in the form of graphs in the paper, raw data is available on request from the corresponding author.

\section{Competing interests}

The authors declare that they have no competing interests.

Received: 17 October 2018 Accepted: 3 July 2019

Published online: 12 July 2019

\section{References}

1. "Ericsson 5G delivers 5 Gbps speeds", Ericsson press release July 2014. Weblink: https://www.ericsson.com/en/press-releases/2014/7/ericsson-5gdelivers-5-gbps-speeds.

2. A Harada et al., $5 \mathrm{G}$ trials with major Global vendors. NTT Docomo Technical Journal, Special articles on 5G Technologies towards 2020 deployment, Volume 17, No. 4, April 2016.

3. P. Fleming, "5G research at Nokia networks", $5 \mathrm{G}$ day at MIT wireless center, May 2015.

4. N. Kaneda, Y. Qian, and T. Itoh, A novel Yagi-Uda dipole array fed by a microstrip-to-CPS transition, in Asia-Pacific Microwave Conf. Dig., Yokohama, Japan, pp. 1413-1416, Dec. 1998.

5. W. R. Deal, N. Kaneda, J. Sor, Y. Qian and T. Itoh, A new quasi-Yagi antenna for planar active antenna arrays, in IEEE Transactions on Microwave Theory and Techniques, vol. 48, no. 6, pp. 910-918, Jun 2000.

6. G. R. DeJean and M. M. Tentzeris, A new high-gain microstrip Yagi array antenna with a high front-to-back (f/b) ratio for wlan and millimeter-wave applications. in IEEE Transactions on Antennas and Propagation, vol. 55, no. 2 pp. 298-304, Feb. 2007.

7. G. R. DeJean, T. T. Thai, S. Nikolaou and M. M. Tentzeris, Design and analysis of microstrip bi-Yagi and quad-Yagi antenna arrays for WLAN applications. in IEEE Antennas and Wireless Propagation Letters, vol. 6, pp. 244-248, 2007.

8. J. Floc'h, A. Ahmad, Broadband QUASI-Yagi antenna for WiFi and WiMax applications. Wirel. Eng. Technol. 4(2), 87-91 (2013)

9. D. Smith, D. Vier, T. Koschny, and C. Soukoulis, Electromagnetic parameter retrieval from inhomogeneous metamaterials. Phys. Rev. E, vol. 71, pp. 036617(1-11), Mar. 2005.

10. D. M. Pozar. Microwave engineering. 4th Edition, Hoboken; John Wiley and Sons Inc., 2011.

11. V. Basavarajappa, B.B. Exposito, L. Cabria and J. Basterrechea, Millimeter wave multi-beam-switching antenna, in Proc. IEEE International Symposium on Wireless Communication Systems (ISWCS), Bologna, Italy, 29-31 Aug. 2017.

12. V. Basavarajappa, B.B. Exposito, L. Cabria and J. Basterrechea, Binary phase controlled multi-beam-switching antenna array for reconfigurable 5G applications. accepted for publication in this special issue of EURASIP JWCN.

13. D.N.Viet, M. D. Renzo, V. Basavarajappa, B. B. Exposito, J. Basterrechea and D. T.P. Huy, Spatial modulation based on reconfigurable antennas: performance evaluation by using the prototype of a reconfigurable antenna. accepted for publication in this special issue of EURASIP JWCN.

\section{Publisher's Note}

Springer Nature remains neutral with regard to jurisdictional claims in published maps and institutional affiliations.

\section{Submit your manuscript to a SpringerOpen ${ }^{\circ}$ journal and benefit from:}

- Convenient online submission

- Rigorous peer review

- Open access: articles freely available online

High visibility within the field

- Retaining the copyright to your article

Submit your next manuscript at $\boldsymbol{\nabla}$ springeropen.com 